\title{
AN IN-VIVO MODEL TO INTERROGATE THE TRANSITION FROM ACUTE TO CHRONIC INFLAMMATION
}

\author{
D. Lickorish ${ }^{1}$, J. Chan ${ }^{1}$, J. Song ${ }^{1}$ and J. E. Davies ${ }^{1,2 *}$ \\ ${ }^{1}$ Institute of Biomaterials and Biomedical Engineering, ${ }^{2}$ Faculty of Dentistry, \\ University of Toronto, Toronto, Ontario, Canada
}

\begin{abstract}
This study describes the modulation of the rodent foreign body giant cell (FBGC) response to subcutaneously implanted, biodegradable poly(lactide-co-glycolide)/ calcium phosphate (PLGA/CaP) composites by application of a thin surface coat of calcium phosphate. Macroporous PLGA/CaP composite scaffolds, with interconnecting macroporosity, were half coated with a $3 \mathrm{~mm}$ thick layer of $\mathrm{CaP}$ by immersion in simulated body fluid. Half-coated scaffolds were implanted subcutaneously in the dorsum of male Wistar rats for 1, 4 and 8 weeks. Specimens were embedded in paraffin and tissue sections evaluated by light microscopy with particular reference to the FBGC response. Histomorphometry revealed that FBGCs were in contact with $6 \%( \pm 3.5 \%)$ of the uncoated half, at 1 week, but no FBGCs were seen on the coated half. By 4 weeks, FBGCs were seen on both the uncoated and coated halves of the scaffolds with $87 \%( \pm 10 \%)$ and $36 \%( \pm 4 \%) \mathrm{FBGC} /$ polymer contact respectively. By 8 weeks these FBGC contact percentages had risen to $97 \%( \pm 0.45 \%)$ in the case of the uncoated halves of scaffolds, but decreased to $22 \%( \pm 4 \%)$ in the case of the $\mathrm{CaP}$-coated halves. Thus the $\mathrm{CaP}$ coating abrogated the FBGC response to the underlying polymer. Such a model may prove useful in providing an experimental system whereby both the mechanisms of biocompatibility and the transition from acute to chronic inflammation could be interrogated.
\end{abstract}

Key words: Polymer scaffold, calcium phosphate, acute inflammation, chronic inflammation, transition from acute to chronic inflammation, foreign body giant cell response, histomorphometry.

* Address for correspondence

J.E. Davies

Institute of Biomaterials and Biomedical Engineering

University of Toronto, 4 Taddle Creek Road

Toronto, Ontario, Canada, M5S 3G9

Telephone Number: (416) 978-1471

FAX Number : (416) 946-5639

E-mail: davies@ecf.utoronto.ca

\section{Introduction}

There is a paucity of information available in the literature to describe the transition between acute and chronic inflammation. While the former is marked by local increased blood volume and leukocyte extravasation, the latter is characterized by the presence of FBGC and fibrous encapsulation (McNally and Anderson, 2002). While much is known about each phenomenon, little is understood about the progression of one to the other. Nevertheless, the mechanisms which govern the choice between resolution of acute inflammation or progression to chronic inflammation are of critical importance in understanding not only the dichotomy between tissue regeneration and repair, but also in understanding the sequelae of the introduction of foreign materials, particularly biodegradable materials, in the body. Yet, little is understood of the cellular mechanisms driving such overt and disparate tissue reactions.

In the oldest of biomaterials applications, sutures, fibrous encapsulation of the tissue-contacting portion of such devices is an advantage both biologically, as the capsule represents an attempt to sequester the foreign body from the systemic environment, and also clinically since it facilitates removal of non-biodegradable sutures after the appropriate postoperative period. For biodegradable suture materials, such as the polyesters, like poly(lactideco-glycolide) (PLGA), the FBGC response ensures a longterm mechanism to eradicate the foreign material from the body. However, in other fields such responses provide a significant obstacle to progress. Thus, in an attempt to reduce the inflammatory response to implanted biosensors, whose function was compromised by fibrous encapsulation, Hickey et al. (2002) loaded biodegradable PLGA microspheres with dexamethasone, a synthetic cortico-steroid with known anti-inflammatory properties. PLGA has also received much attention as a delivery vehicle for vaccines where the generation of an acute inflammatory response is essential to the recruitment of dendritic cells, derived from the monocyte lineage, which act as antigen-presenting cells to trigger the required host immune response to the delivered vaccine (Paglia and Colombo, 2002). However, the transition between this beneficial aspect of acute inflammation to a chronic inflammatory response to the PLGA which, with appearance of FBGCs, compromises the efficacy of dendritic cell antigen presentation, remains a hurdle to be overcome (Babensee et al., 2002). Such biodegradable polyesters are, of course, widely employed in the biomaterials and tissue engineering fields where, again, their chronic degradation as a function of giant cell activity 
is essential in many applications such as bone plates and screws, but may be a disadvantage in tissue engineering scaffolds where polymer degradation products may have an adverse effect on the invasion of host tissue into the cell/polymer construct (Agrawal and Ray, 2001).

Since FBGCs are the fusion products of monocytic precursors, which are also the precursors to macrophages and dendritic cells, the presence of such leukocytes in the wound healing compartment may be of central importance in driving the tissue reaction to the material (Brodbeck et al., 2002; Matheson et al., 2004). Indeed, there is considerable evidence to illustrate the central role played by such cells. For example, Huitinga et al. (1995) showed, using a T-cell-dependent autoimmune model of encephalomyelitis, that infiltrating macrophages were crucial effectors of tissue injury and Jun et al. (1999) showed that macrophage depletion blocked disease in an animal model of autoimmune diabetes. These and other examples have been recently, and comprehensively, reviewed by Duffield (2003). In addition, Tang et al. (1996) reported that phagocyte accumulation on implant surfaces could be abrogated by administration of recombinant neutrophil inhibitory factor that blocks the CD11bfibrinogen interaction. Thus, while leukocyte extravasation and interaction with an implanted material are hallmarks of acute inflammation, the appearance of FBGCs would seem to be indicative of the transition from the acute to the chronic inflammatory phenomenon, without negating the possibility, as is the case with the tissue reaction to some polymeric materials, that both acute and chronic reactions may co-exist.

Such would not seem to be the case with another broad class of biomaterials, the calcium phosphates, which have long been considered biocompatible and, in general, not instigators of a FBGC response upon implantation. There are notable exceptions to this generalized statement both from the biomaterials and pathology literatures where $\mathrm{CaP}$ particulates have been reported to generate giant cell responses and $\mathrm{CaPs}$ are known to be a common cause of crystal-induced joint arthropathies respectively (O'Shea and McCarthy, 2004). Nevertheless, CaP lithomorphs are not reported to induce a FBGC response and, although macrophage accumulation at the interface with such biomaterials must occur during the acute inflammatory phase of healing, this would not seem to transition into a FBGC response.

Given the exposure of these important gaps in our knowledge concerning the transition between acute and chronic inflammation, we report here some preliminary observations made during experiments designed to test the efficacy of a CaP coating of a biodegradable composite tissue engineering scaffold material comprising PLGA and a CaP particulate. We emphasize that the reported observations were not the reason for the original experiment, but are of sufficient interest to suggest this approach as a potential model system to interrogate the cellular and molecular mechanisms underlying the transition from acute to chronic inflammation which we believe, given the arguments presented above, may be of some interest to those working in several otherwise unconnected fields of research.
In brief, we implanted biodegradable tissue engineering scaffolds subcutaneously in rats. Half of each scaffold had been coated with a thin $\mathrm{CaP}$ layer that, we found, abrogated the FBGC response seen on the uncoated scaffold surface. We show that the spatial delineation of the disparate cellular responses was closely related to change in surface of the scaffold material. As such, this model may prove useful in not only exploring the mechanisms of the FBGC response as suggested above, but also deconvoluting the structure/function relationships underlying the biocompatibility of $\mathrm{CaP}$ materials.

\section{Materials and Methods \\ Polymeric scaffolds}

Macroporous PLGA $(75: 25)$ scaffolds were prepared as previously described (Guan and Davies, 2004). Briefly, PLGA (Birmingham Polymers, Birmingham, AL, USA) was dissolved in dimethylsulfoxide and thoroughly mixed with $\mathrm{CaP}$ particles. The resulting PLGA/CaP mixture was poured into a mold filled with fused sugar (glucose) crystals (grain size of $\sim 0.85-1.18 \mathrm{~mm}$ ) and transferred to a fridge at $-18^{\circ} \mathrm{C}$ for $1 \mathrm{hr}$ to set. The sugar crystals were purchased form Redpath Sugar (Toronto, Ontario, Canada). The PLGA was precipitated and sugar crystals leached out by immersion in double distilled $\mathrm{H}_{2} \mathrm{O}\left(\mathrm{ddH}_{2} \mathrm{O}\right)$ at room temperature $\left(20^{\circ} \mathrm{C}\right)$ for 3 days, during which time the $\mathrm{ddH}_{2} \mathrm{O}$ was changed approximately 4 times per day.

\section{Construct preparation}

Discs measuring $10 \mathrm{~mm}$ in diameter and $2 \mathrm{~mm}$ thickness were cut from a single $10 \mathrm{~cm} \times 10 \mathrm{~cm} \times 1 \mathrm{~cm}$ PLGA/CaP block using a stainless steel hole saw attached to a highspeed Dremel drill (Model 398) and drill press (Model 212, type II) (Dremel, Robert Bosch Tool Corporation, Mount Prospect, IL, USA) (Karp et al., 2004). To provide physical support to the macroporous material following implantation, a perspex chamber ring (Millipore PR00 014 00) (Millipore, Billerica, MA, USA) was used to contain each scaffold. To ensure the scaffold remained within the ring, a nylon strut (Millipore PR 00000 00) was solvent welded on each side of the ring using Millipore cement (XX70 000 72) (Fig. 1). We refer to this protective construct as a "cage".

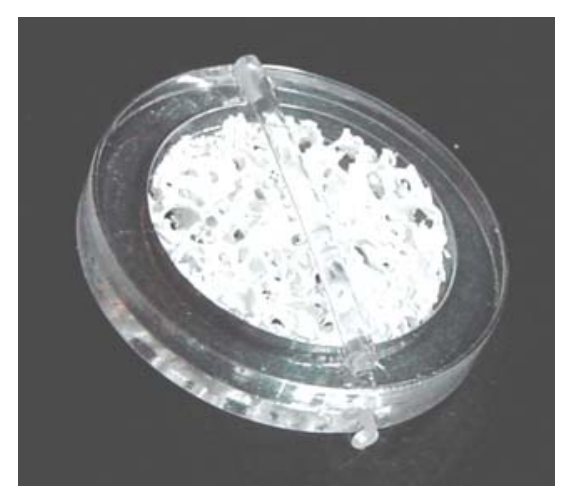

Figure 1. Macroporous PLGA/CaP scaffold within a Millipore Plexiglas ${ }^{\circledR}$ diffusion chamber ring (internal diameter $10 \mathrm{~mm}$ ) with nylon protector bar. 


\section{CaP coating of PLGA}

In addition to the $\mathrm{CaP}$ phase embedded within the polymeric structure, a thin surface layer of $\mathrm{CaP}$ was deposited onto half of the scaffolds using the following solution chemistry technique. Samples to be coated were pre-wetted in $70 \%$ ethanol, and rinsed in deionised water. Half of the scaffold was immersed in concentrated [x 5] simulated body fluid (SBF: $\mathrm{Na}^{+} 142.0 \mathrm{mM}, \mathrm{K}^{+} 5.0 \mathrm{mM}, \mathrm{Mg}^{2+} 1.5 \mathrm{mM}, \mathrm{Ca}^{2+} 2.5 \mathrm{mM}$, $\mathrm{Cl}^{-} 147.8 \mathrm{mM}, \mathrm{HCO}^{3-} 4.2 \mathrm{mM}, \mathrm{HPO}_{4}{ }^{2-} 1.0 \mathrm{mM}, \mathrm{SO}_{4}{ }^{2-} 0.5 \mathrm{mM}$ ) and maintained at $37^{\circ} \mathrm{C}$ for a period of 5 days, with daily changes of SBF (Barrere et al., 2002).

\section{Scanning electron microscopy (SEM)}

SEM was used to examine the microstructure of the coated scaffold and to ensure that only half of the scaffold had been coated with $\mathrm{CaP}$. Following immersion in SBF, samples were rinsed in $\mathrm{ddH}_{2} \mathrm{O}$, air dried, coated with platinum and examined using a Hitachi S2500 SEM (Hitachi, Tokyo, Japan).

\section{Subcutaneous implantation}

The following implantation protocol was reviewed and approved by the Animal Ethics Committee of the University of Toronto. Nine male Wistar rats, weighing approximately $200 \mathrm{~g}$, were anaesthetized using Isofluorane. An incision was made in the dorsal cutis and a subcutaneous pocket was created using blunt dissection. A half coated scaffold, contained within a cage, was placed in this pocket and the overlying skin closed using staples. Constructs were left insitu for 1,4 and 8 weeks ( $n=3$ per time point). Animals received postoperative analgesia via subcutaneous administration of buprenorphine $(0.05 \mathrm{mg} / \mathrm{kg})$ and were left to recover under a heat lamp. Following recovery, animals received food and water ad libitum.

\section{Histological preparation}

Following euthanasia, constructs with surrounding soft tissue were removed and fixed in formalin for $48 \mathrm{hrs}$. The nylon struts were fractured at the solvent weld points and pulled out of the tissue. Then, using a razor blade, the surrounding tissue was separated from the outer perimeter of the Perspex ring and the scaffold and related tissue removed from the ring. The scaffold/tissue specimen was then bisected and embedded in paraffin. Sections, $6 \mathrm{~mm}$ thick, were cut from the paraffin blocks, were stained with haematoxylin and eosin and qualitatively examined using a light microscope (Olympus BX51) (Olympus, Melville, NY, USA) interfaced with a digital camera (SPOT RT, Digital Instruments; now Veeco Instruments, Woodbury, NY, USA) running SPOT software. Particular attention was paid to the number and distribution of FBGCs.

\section{Quantitative analysis}

To facilitate measurement of the FBGC response, light microscopic digital images were acquired using a $10 \mathrm{x}$ objective lens. An average of eight micrographs were taken for each slide to ensure coverage of the entire tissue section. Lines were traced around the contact surfaces of PLGA with repair tissue on each image using Adobe Photoshop (Adobe Inc., San Jose, CA, USA). Green lines were used to represent the surfaces of PLGA polymer that were free of FBGC and red lines used to represent the areas where FBGCs were in direct contact with the polymer (Fig. 2). Following determination of FBGC contact surface, Image J software (http://rsb.info.nih.gov/ij/) was used to convert the contact surface measurement into a FBGC linear contact percentage. Data was expressed as mean \pm standard deviation ( $\mathrm{n}=3$ for each time point).

\section{Results \\ Scanning electron microscopy}

SEM revealed a confluent crystalline layer, approximately $3 \mu \mathrm{m}$ thick, on the part of the scaffold immersed in SBF. Imaging the junction between the uncoated and coated areas demonstrated that the material had been successfully half coated; although the junction between the coated and uncoated halves was imperfect since some sites of calcium phosphate nucleation extended randomly to a distance of about $50 \mu \mathrm{m}$ from the solid boundary (Fig. 3).

\section{Histological observations}

1 week (not shown). By one week, a loose, fibrovascular tissue had penetrated throughout the pores of all constructs on both coated and uncoated sides of the scaffolds. Small numbers of FBGCs were identified in the tissue adjacent to the uncoated side but these were not a prominent histological feature. A similar tissue response was seen in the coated half, however no FBGCs were observed.

4 weeks. At low magnification the stark contrast in tissue response between the two halves of the scaffold could be observed (Fig. 4). The uncoated portion of the scaffolds had provoked a robust FBGC response. Numerous FBGCs were observed in dense collagenous tissue adjacent to the degrading polymer, lining the interface between tissue and polymer (Fig. 5a). In sharp contrast, the coated portion of the scaffolds showed little accumulation of FBGC either deep to, or in direct contact with, the $\mathrm{CaP}$ coating (Fig. 5b).

8 weeks. By 8 weeks the uncoated polymer had been extensively degraded resulting in fragmentation of the polymer into numerous sites, each surrounded by multiple FBGCs (Fig. 6a). In contrast, the CaP coated half had not fragmented and retained its open pore structure. Occasional FBGCs were observed adjacent to the CaP coating (Fig. 6b).

\section{Histomorphometry}

The FBGC/polymer contact at 1 week was approximately $6 \%( \pm 3.5 \%)$ on the uncoated half, and zero on the coated half (Fig. 7). By 4 weeks this had increased on both the uncoated and coated halves to $87 \%( \pm 10 \%)$ and $36 \%$ $( \pm 4 \%$ ) respectively. At 8 weeks, the FBGC contact with the uncoated half had increased again to $97 \%( \pm 0.45 \%)$, while that on the coated half had decreased to $22 \%( \pm 4 \%)$. 

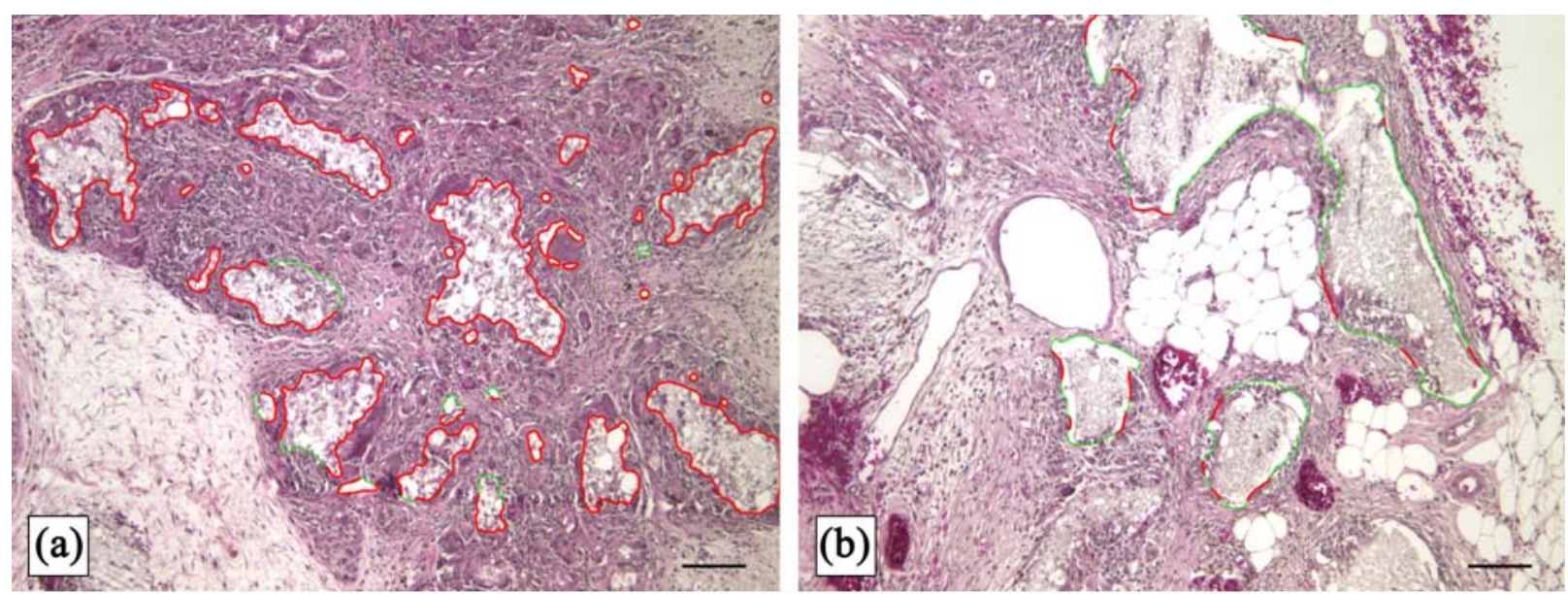

Figure 2. Light micrographs of (a) Uncoated Half of scaffold Bar $=100 \mu \mathrm{m}$ (b) Coated half of scaffold Bar = $100 \mu \mathrm{m}$. Green lines indicate no FBGC contact with polymer whilst red lines indicate FBGC contact with polymer at 4 weeks.
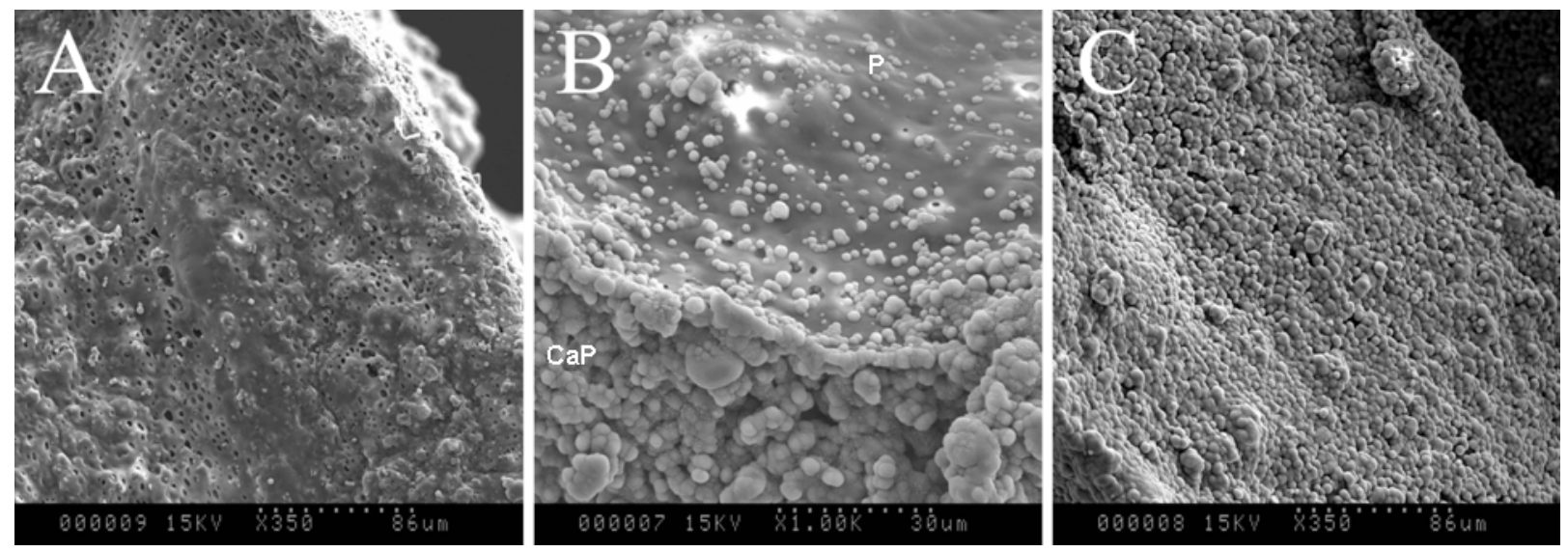

Figure 3. Scanning electron micrographs of scaffold showing (a) part of the uncoated half of polymer. Note: the polymer surface, which is microporous, is also rough as a result of the inclusion of the CaP particulate phase. Field Width $(\mathrm{FW})=290 \mu \mathrm{m}$; (b) junction between the CaP coated and uncoated central region of the scaffold. Note: At bottom of the field of view the CaP coating is complete, but some nucleated CaP deposits extend to about $50 \mu \mathrm{m}$ beyond this solid junction onto the remaining polymer surface (P - above) $\mathrm{FW}=100 \mu \mathrm{m}$; (c) fully coated half of polymer. FW $=290 \mu \mathrm{m}$. Note: In (b) and (c), the CaP coating exhibits a globular appearance and thus introduces a change in surface topography with respect to the uncoated polymer.

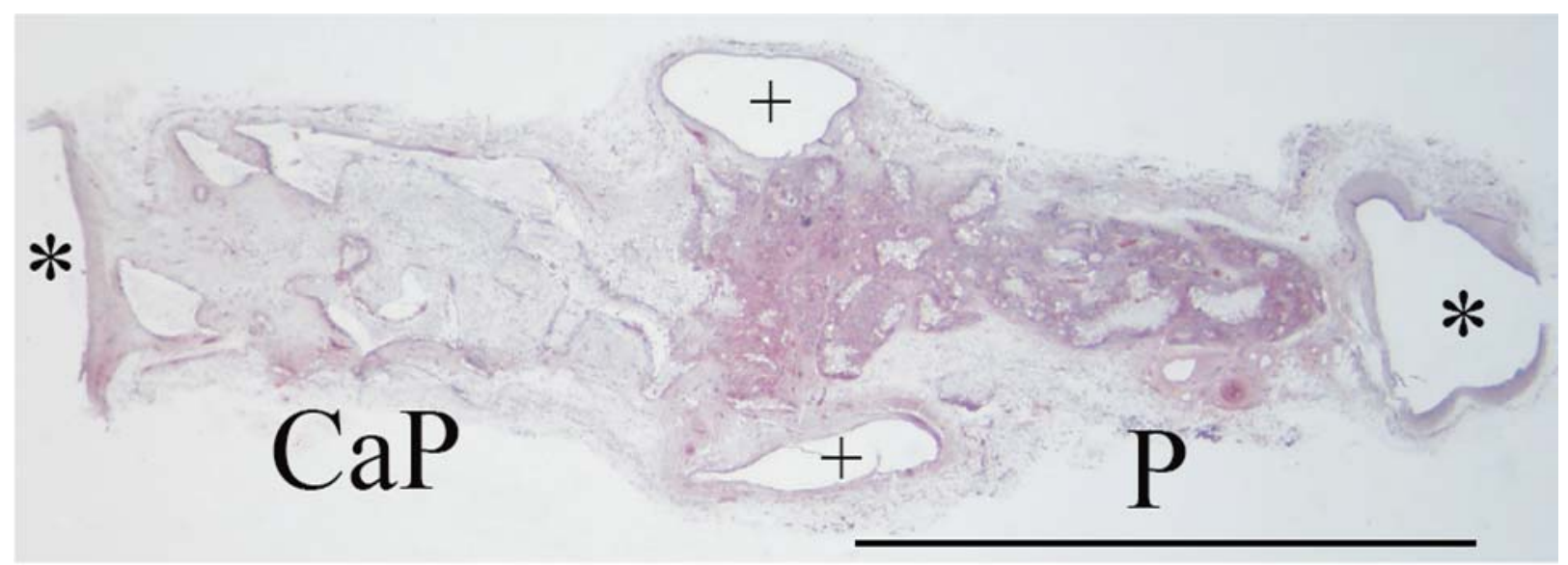

Figure 4. Low magnification image obtained using a stereo dissecting microscope of a complete cross section of an embedded scaffold and "cage" after 4 wks implantation. The empty areas $(*)$ at right and left were those filled by the diffusion chamber ring, while those $(+)$ top and bottom represent the cross-sections of the nylon protective bars. The scaffold has been bisected, within the "cage" so that the left half is the coated half (CaP) and the right half is uncoated $(\mathrm{P})$. Note: the contrast in tissue response between the coated and uncoated halves is easily visible at this magnification, as is the apparent increase in number of scaffold fragments in the uncoated half. Haematoxylin and eosin. Bar $=5 \mathrm{~mm}$. 

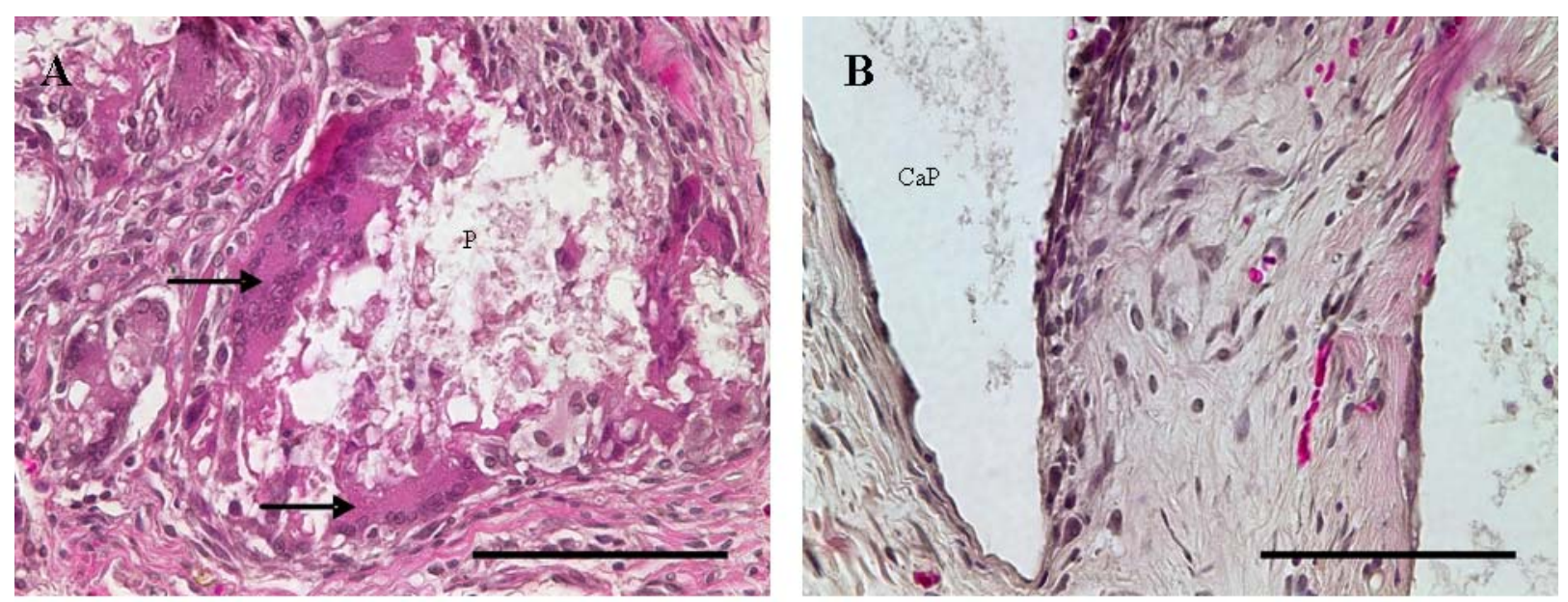

Figure 5. (a) Light micrograph of uncoated half of scaffold at 4 wks showing large multinucleated FBGCs (arrows) and degrading polymer (p). Note: the staining with the remaining polymer is indicative of polymer degradation - compare with Figure 6a. Haematoxylin and eosin. Bar $=100 \mu \mathrm{m}$. (b) Light micrograph of coated half of scaffold at $4 \mathrm{wks}$. Note the absence of FBGCs and the quiescent nature of the fibrovascular repair tissue in contact with the coated polymer $(\mathrm{CaP})$. Also, in contrast to Figure 5a, there is an absence of staining in the area previously occupied by the scaffold. This changed by 8 weeks - see Figure 6 . Some occasional giant cells were found in other fields of view. Haematoxylin and eosin. Bar $=100 \mu \mathrm{m}$.
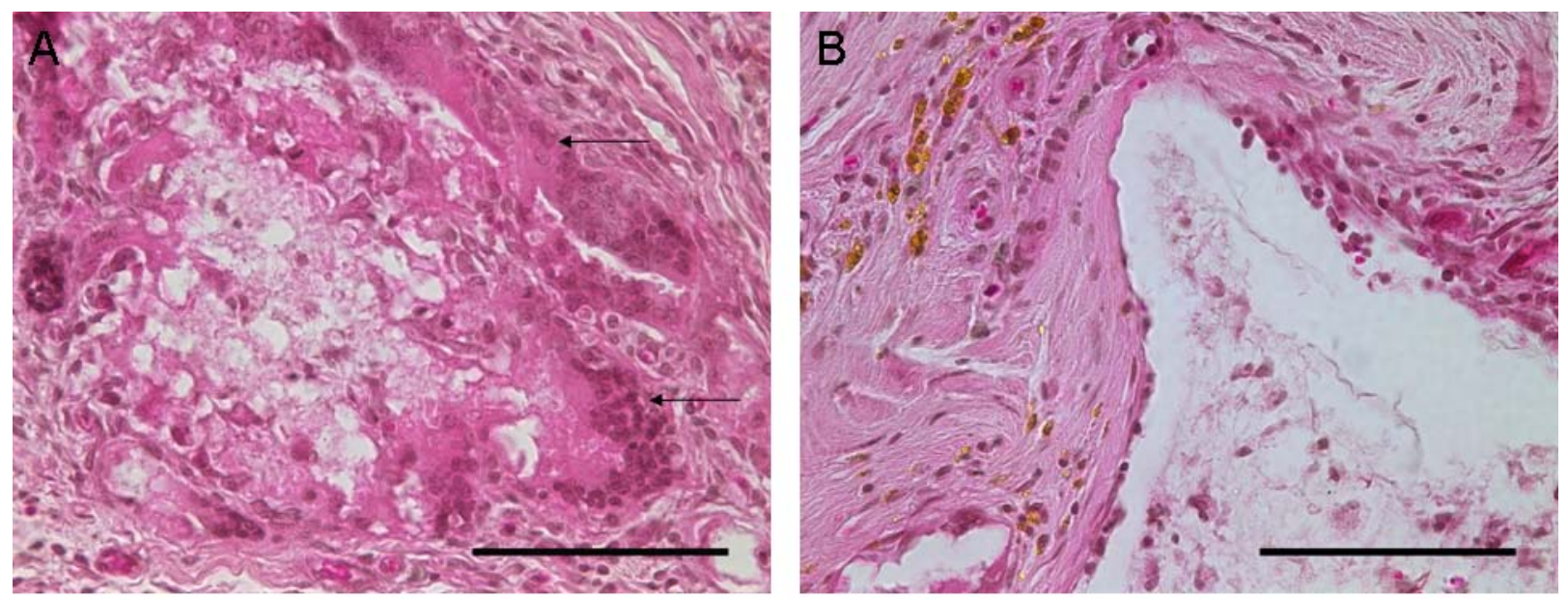

Figure 6. (a) Light micrograph of uncoated half of scaffold at 8 wks. At this time point the uncoated half of the scaffold was fragmented and was surrounded by numerous FBGCs (arrows). The remaining polymer (illustrated by white space in this section) is negligible since most the space previously occupied by the polymer is stained tissue. Haematoxylin and eosin. Bar $=100 \mu \mathrm{m}$ (b) Light micrograph of coated half of scaffold at $8 \mathrm{wks}$.

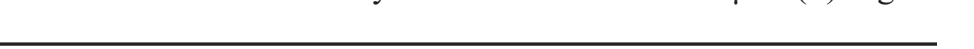

FBGC Contact Surface Area

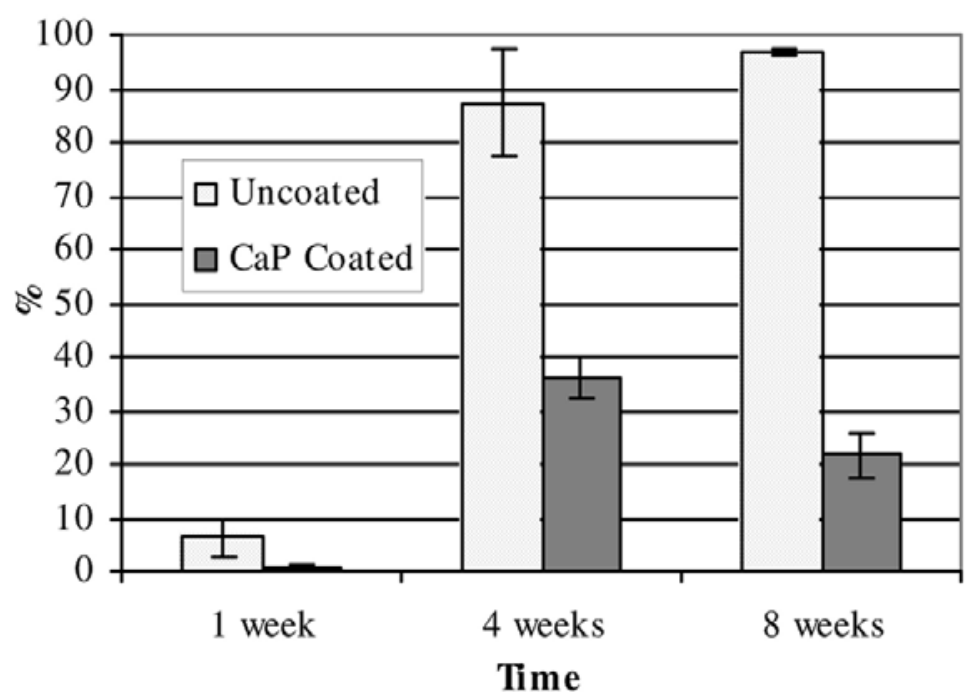

The macroporosity of the scaffold was maintained and FBGCs were reduced in number compared to $4 \mathrm{wks}$. More staining is now evident within the polymer, compared to Figure $5 b$, indicating polymer degradation has occurred, yet the surrounding tissue is fibro-vascular in nature rather than occupied by giant cells as seen in Figures $5 \mathrm{a}$ and $6 \mathrm{a}$. Haematoxylin and eosin. Bar = $100 \mu \mathrm{m}$.

Figure 7. Graph relating the FBGC contact percentage with scaffold surface over time. Data is mean \pm standard deviation. $n$ $=3$ for each time point. 


\section{Discussion}

Our results show that a simple $3 \mu \mathrm{m}$ thick surface coating of $\mathrm{CaP}$ (Lickorish et al., 2004) can abrogate the rodent FBGC response to an implanted PLGA/CaP scaffold. This host response was sharply contrasted on the opposing sides of each scaffold, and the spatial delineation of the disparate cellular responses was closely related to change in surface of the scaffold material. Thus, while the acute inflammatory response to the uncoated surface transitioned to a chronic inflammation with FBGCs in contact with $97 \%$ of the available scaffold surface by 8 weeks, the FBGC response to the coated side was significantly less at 4 weeks and, unlike that to the uncoated side, decreased to $22 \%$ by 8 weeks. This dramatic difference in host reaction within the same biological system may provide an in-vivo model by which mechanisms of inflammatory biology may be investigated.

The ability to control the inflammatory response holds great significance for those attempting to introduce foreign materials into the body, whether these materials be biodegradable polymers for tissue engineering purposes, biosensors to monitor physiological fluctuations, vaccines designed to generate an immune response or orthopaedic devices used in arthroplasty. Clearly, implantation of a foreign material within the body will provoke an acute inflammation; an unavoidable consequence of tissue injury. However, what is unclear, and largely overlooked, are the mechanisms by which this ordinarily transient phenomenon fails to resolve, leading to the development of a chronic inflammation that is widely described as detrimental to biomaterial function (Anderson, 1988). Polymers have proven popular materials from which to fabricate implantable devices for a wide range of tissue engineering applications. More specifically, polyesters such as polyglycolic acid (PGA), polylactic acid (PLA) and their co-polymer, PLGA, feature prominently as resorbable fracture fixation plates in the orthopaedic field (Eppley, 1998), as microparticles for introduction of vaccines and drugs and as resorbable porous scaffolds for cell delivery (Chandrashekar and Udupa, 1996; Kempen et al., 2004). Whilst much of the literature describes biodegradable polyester materials as being biocompatible, there are reports that implantation of these materials may result in a chronic inflammatory response (Solheim et al., 2000; Bostman and Pihlajamaki, 2000). Little data is available on how this phenomenon is mediated, but chronic inflammation is often attributed to the irritant effect of a local reduction in $\mathrm{pH}$, due to acidic polymeric degradation products (Agrawal and Athanasiou, 1997).

$\mathrm{CaP}$ has a well documented history as a biocompatible material and continues to be a successful clinical adjunct, even replacement, to the bone graft in orthopaedic procedures. But, the reason for this biocompatibility, indeed, even the mechanism through which $\mathrm{CaP}$ may modulate the inflammatory response, is also similarly poorly understood. In an orthopaedic context, the coating of materials with $\mathrm{CaP}$ has generally been performed to improve the osteoconductive properties of the material being coated. Using SBF, materials ranging in composition from metallic, ceramic to polymeric (Tanahashi et al., 1995; Lickorish et al., 2004; Barrere et al., 1999, Leng et al., 2003) have been coated with a thin layer of $\mathrm{CaP}$ and chronic inflammation has not been reported following in-vivo implantation of these materials. There are several possible mechanisms by which CaP could influence the FBGC response. These include, but are not limited to, effects of $\mathrm{CaP}$ on surface protein adsorption and monocyte/ macrophage attachment, proliferation, and fusion.

\section{The role of protein adsorption}

The adsorption of proteins, principally those of the complement and coagulation cascades, to a biomaterial surface following implantation into the body is thought to play a significant role in the subsequent tissue response (Janatova, 2000). The species and conformation of adsorbed proteins present on a biomaterial may influence monocyte adhesion, subsequent differentiation and proliferation of macrophages on the implant surface, (Tang et al., 1996; Hu et al., 2001) and may even be responsible for encouraging macrophage fusion into FBGCs. Few data are available on the specific protein species that adsorb preferentially onto PLGA versus CaP. Indeed, this has been notoriously difficult to study (Collier et al., 2004). Nevertheless, it is not unreasonable to speculate that the sharp delineation between tissue response to the $\mathrm{CaP}$ and PLGA, observed in the present study, could be a function of adsorbed proteins attributed to the differing surface chemistry and/or topography (Jenney and Anderson, 2000). Since a CaP coating reduces the likelihood for formation of the FBGC, this offers an attractive alternative antigen delivery vehicle, as $\mathrm{CaP}$ coated microspheres, to dendritic cells, especially if the antigen could be incorporated within the coating to maintain dose rates over time as the $\mathrm{CaP}$ is resorbed.

\section{The role of macrophages}

Manipulation of host protein layer by surface functionalization or modification is a popular method to promote/inhibit cell interactions with implanted materials (DeFife et al., 1999; Healy 1999; Collier and Anderson, 2002). Hu et al. (2001) reported the involvement of adsorbed fibrinogen as playing a key role in the attraction of phagocytic cells to the surfaces of implanted materials. Indeed, macrophages play a pivotal role in the inflammatory response (Anderson and Miller, 1984). Appropriately, strategies designed to modulate inflammation following biomaterial implantation attempt to directly influence the interaction of the macrophage with the biomaterial, reasoning that if a surface is sufficiently inhospitable to monocyte adhesion then subsequent macrophage maturation and FBGC formation is unlikely to follow (Hamilton 2003; Collier et al., 2004). Such strategies have included incorporation of non steroidal antiinflammatory agents within polymer microspheres (Hickey et al., 2002). Kao and Lee (2001) describe the importance of the RGD and PHSRN regions of the fibronectin molecule in modulating the host response, macrophage adhesion and FBGC formation in-vivo. Incorporation of vitamin $\mathrm{E}$ onto a polyurethane biomaterial resulted in 
reduced FBGC-mediated degradation (Schubert et al., 1996). Iwasaki et al. (2002) blended 2-methacryloyloxyethyl phosphorylcholine (MPC) with PLGA to reduce the inflammatory reaction of adherent cells whilst Khouw et al. (1998) used antibodies against interferon gamma, a $\mathrm{T}$ cell derived cytokine known to activate macrophages, to delay the formation and function of FBGC. Clearly, the numerous ways by which macrophagebiomaterial interactions can be affected underscores the complexity of the host response.

\section{Macrophage fusion}

Once macrophages have adhered to a surface, the molecular mechanism describing their fusion to form FBGC is also unclear (Matheson et al., 2004) though macrophage fusion receptor, CD44 and CD47 have been reported as playing a role in promoting fusion (Vignery, 2000). Cytokines have also been implicated in fusion as the inability of macrophages to phagocytose large amounts of material, so called 'frustrated phagocytosis' (Matzelle and Babensee, 2004), may encourage production of proinflammatory mediators to recruit assistance. IL-4 and IL13 have been shown to induce FBGC formation in-vitro (McNally and Anderson, 2002). Such could encourage progression to a chronic inflammation in-vivo if there is a shift in the equilibrium between pro- and anti-inflammatory chemokines. Furthermore, it has been reported that macrophages greater than 8 days old (Most et al., 1997), or less than a certain 'critical density' (Vignery, 2000), show almost no fusion into FBGCs, the former highlighting the importance of unravelling the temporal nature of macrophage interaction with a material and the latter supporting the underlying rationale for attempting to modulate initial monocyte adhesion to the biomaterial.

\section{Concluding remarks}

In this preliminary study we have rendered a macroporous PLGA/CaP scaffold 'anti-inflammatory', by simply incorporating a thin surface film of $\mathrm{CaP}$. The significance of this brief study lies, firstly, in the fabrication of a material that can modulate the inflammatory response and secondly, in describing an in-vivo model that enables us to address questions regarding the transition from acute to chronic inflammation. The inflammatory response involves a complex interplay between a myriad of signaling molecules, produced by and intended for, a wide variety of cells, and it is clear that to engineer the requisite level of control over this process, a greater appreciation of the basic biology involved is needed. Thus, this novel experimental approach will enable further interrogation of the mechanisms of acute and chronic inflammation. Moreover, in addition to illuminating the mechanisms governing the progression from acute to chronic inflammation, this model will enable the mechanisms of polyester and $\mathrm{CaP}$ biocompatibility to be further dissected. This approach may encourage dialogue between unconnected fields which, despite divergent applications, share a similar interest in understanding and controlling the adverse inflammatory response.

\section{Acknowledgements}

The authors would like to thank Susan Carter for assistance with animal surgeries. We would also like to acknowledge the financial support provided by an Ontario Research and Development Challenge Fund (ORDCF) grant to JED and BoneTec Corporation, Toronto.

\section{References}

Agrawal CM, Athanasiou KA (1997) Technique to control $\mathrm{pH}$ in vicinity of biodegrading PLA-PGA implants. J Biomed Mater Res 38: 105-114.

Agrawal CM, Ray RB (2001) Biodegradable polymeric scaffolds for musculoskeletal tissue engineering. J Biomed Mater Res 55: 141-150.

Anderson JM (1988) Inflammatory response to implants. ASAIO Trans 34 :101-107.

Anderson JM, Miller KM (1984) Biomaterial biocompatibility and the macrophage. Biomaterials 5: 510

Babensee JE, Stein MM, Moore LK (2002) Interconnections between inflammatory and immune responses in tissue engineering. Ann NY Acad Sci 961: 360-363.

Barrere F, van Blitterswijk CA, Groot K, Layrolle P (2002) Influence of ionic strength and carbonate on the $\mathrm{Ca}-\mathrm{P}$ coating formation from $\mathrm{SBF} \times 5$ solution. Biomaterials 23: 1921-1930.

Barrere F, Layrolle P, van Blitterswijk CA, de Groot K (1999) Biomimetic calcium phosphate coatings on Ti6AI4V: a crystal growth study of octacalcium phosphate and inhibition by $\mathrm{Mg}^{2+}$ and $\mathrm{HCO}_{3}^{-}$. Bone 25(2 Suppl): 107S-111S.

Bostman O, Pihlajamaki H (2000) Clinical biocompatibility of biodegradable orthopaedic implants for internal fixation: a review. Biomaterials 21: 2615-2621.

Brodbeck WG, Nakayama Y, Matsuda T, Colton E, Ziats NP, Anderson JM (2002) Biomaterial surface chemistry dictates adherent monocyte/macrophage cytokine expression in vitro. Cytokine 18: 311-319.

Chandrashekar G, Udupa N (1996) Biodegradable injectable implant systems for long term drug delivery using poly(lactic-co-glycolic) acid copolymers. J Pharm Pharmacol 48: 669-674.

Collier TO, Anderson JM (2002) Protein and surface effects on monocyte and macrophage adhesion, maturation, and survival. J Biomed Mater Res 60: 487-496.

Collier TO, Anderson JM, Brodbeck WG, Barber T, Healy KE (2004) Inhibition of macrophage development and foreign body giant cell formation by hydrophilic interpenetrating polymer network. J Biomed Mater Res 69: 644-650 .

DeFife KM, Jenney CR, Colton E, Anderson JM (1999) Disruption of filamentous actin inhibits human macrophage fusion. FASEB J 13: 823-832.

Duffield JS (2003) The inflammatory macrophage: a story of Jekyll and Hyde. Clin Sci (Lond) 104: 27-38. 
Eppley BL (1998) Use of a resorbable fixation technique for maxillary fractures. J Craniofac Surg 9: 317 321.

Guan L, Davies JE (2004) Preparation and Characterization of a highly macroporous biodegradeable composite scaffold for bone engineering applications. J Biomed Mater Res, in press.

Hamilton JA (2003) Nondisposable materials, chronic inflammation, and adjuvant action. J Leukoc Biol 73: $702-$ 712.

Healy KE, Rezania A, Stile RA (1999) Designing biomaterials to direct biological responses. Ann NY Acad Sci 875: 24-35.

Hickey T, Kreutzer D, Burgess DJ, Moussy F (2002) In vivo evaluation of a dexamethasone/ PLGA microsphere system designed to suppress the inflammatory tissue response to implantable medical devices. J Biomed Mater Res 61: 180-187.

Hu WJ, Eaton JW, Ugarova TP, Tang L (2001) Molecular basis of biomaterial-mediated foreign body reactions. Blood 98: 1231-1238.

Huitinga I, Ruuls SR, Jung S, Van Rooijen N, Hartung HP, Dijkstra CD (1995) Macrophages in T cell linemediated, demyelinating, and chronic relapsing experimental autoimmune encephalomyelitis in Lewis rats. Clin Exp Immunol 100: 344-351.

Iwasaki Y, Sawada S, Ishihara K, Khang G, Lee HB (2002) Reduction of surface-induced inflammatory reaction on PLGA/MPC polymer blend. Biomaterials 23: 3897-3903.

Janatova J (2000) Activation and control of complement, inflammation, and infection associated with the use of biomedical polymers. ASAIO J 46: S53-62.

Jenney CR, Anderson JM (2000) Adsorbed serum proteins responsible for surface dependent human macrophage behavior. J Biomed Mater Res 49: 435-447.

Jun HS, Yoon CS, Zbytnuik L, van Rooijen N, Yoon JW (1999) The role of macrophages in T cell-mediated autoimmune diabetes in nonobese diabetic mice. J Exp Med 189: 347-358.

Kao WJ, Lee D (2001) In vivo modulation of host response and macrophage behavior by polymer networks grafted with fibronectin-derived biomimetic oligopeptides: the role of RGD and PHSRN domains. Biomaterials 22: 2901-2909.

Karp JM, Rzeszutek K, Shoichet MS, Davies JE (2003) Fabrication of precise cylindrical three-dimensional tissue engineering scaffolds for in vitro and in vivo bone engineering applications. J Craniofac Surg 14: 317-323.

Kempen DH, Lu L, Zhu X, Kim C, Jabbari E, Dhert WJ, Currier BL, Yaszemski MJ (2004) Development of biodegradable poly(propylene fumarate)/poly(lactic-coglycolic acid) blend microspheres. II. Controlled drug release and microsphere degradation. J Biomed Mater Res 70: 293-302.

Khouw IM, van Wachem PB, de Leij LF, van Luyn MJ (1998) Inhibition of the tissue reaction to a biodegradable biomaterial by monoclonal antibodies to IFN-gamma. J Biomed Mater Res 41: 202-210.
Leng Y, Chen J, Qu S (2003) TEM study of calcium phosphate precipitation on HA/TCP ceramics. Biomaterials 24: 2125-2131.

Lickorish D, Guan L, Davies, JE (2004) Calcium phosphate coating of macroporous polymer-ceramic scaffolds: effect of coating on foreign body giant cell response. Proc $7^{\text {th }}$ World Biomaterials Congress Sydney, Abstr \#1780. Australian Society for Biomaterials Inc., Victoria, Australia. ISBN 1877040193.

Lickorish D, Ramshaw JA, Werkmeister JA, Glattauer V, Howlett CR (2004) Collagen-hydroxyapatite composite prepared by biomimetic process. J Biomed Mater Res 68: 19-27.

Matheson MA, Santerre JP, Labow RS (2004) Changes in macrophage function and morphology due to biomedical polyurethane surfaces undergoing biodegradation. J Cell Physiol 199: 8-19.

Matzelle MM, Babensee JE (2004) Humoral immune responses to model antigen co-delivered with biomaterials used in tissue engineering. Biomaterials 25: 295-304.

McNally AK, Anderson JM (2002) Beta1 and beta2 integrins mediate adhesion during macrophage fusion and multinucleated foreign body giant cell formation. Am J Pathol 160: 621-630.

Most J, Spotl L, Mayr G, Gasser A, Sarti A, Dierich MP (1997) Formation of multinucleated giant cells in vitro is dependent on the stage of monocyte to macrophage maturation. Blood 89: 662-671.

O'Shea FD, McCarthy GM (2004) Basic calcium phosphate deposition in the joint: a potential therapeutic target in osteoarthritis. Curr Opin Rheumatol 16: 273-278.

Paglia P, Colombo MP (2002) Macrophages as antigenpresenting cells: Relationship to dendritic cells and use in vaccination studies. In: The Macrophage. Chapter 3. Burke B, Lewis CE (eds). Oxford University Press, Oxford. pp 103-137.

Schubert MA, Wiggins MJ, DeFife KM, Hiltner A, Anderson JM (1996) Vitamin E as an antioxidant for poly(etherurethane urea): in vivo studies. J Biomed Mater Res 32: 493-504.

Solheim E, Sudmann B, Bang G, Sudmann EJ (2000) Biocompatibility and effect on osteogenesis of poly(ortho ester) compared to poly(DL-lactic acid) J Biomed Mater Res 49: 257-263.

Tanahashi M, Yao T, Kokubo T, Minoda M, Miyamoto T, Nakamura T, Yamamuro T (1995) Apatite coated on organic polymers by biomimetic process: improvement in its adhesion to substrate by glow-discharge treatment. J Biomed Mater Res 29: 349-357.

Tang L, Ugarova TP, Plow EF, Eaton JW (1996) Molecular determinants of acute inflammatory responses to biomaterials. J Clin Invest 97: 1329-1334.

Vignery A (2000) Osteoclasts and giant cells: macrophage-macrophage fusion mechanism. Int J Exp Pathol 81: 291-304. 


\section{Discussion with Reviewers}

J. Kirkpatrick: The authors quite rightly indicate that the differences in tissue response could be a result of differing types of protein adsorption, this in turn being governed by surface chemistry and/or topography. The calcium phosphate coating clearly altered the topography of the implant surface. Is it possible to modulate this surface topography to give a topography similar to the uncoated composite? If so, this would enable the authors (in a further experimental study) to delineate between the roles of surface chemistry and topography in determining the differences in tissue response.

Authors: The quick answer is "No" although we would agree that deconvoluting the respective roles of surface chemistry and topography are of great importance in this type of study. Unfortunately, to design such experimental substrates is not trivial and thus samples of identical topography, but different chemistry, are normally unattainable. Surface grown calcium phosphate layers, as we have employed herein comprise small crystallites of, generally, leaflet form. Other calcium phosphate deposition techniques, such as organic sol-gel coatings, result in highly smooth surfaces. However, the latter involve high temperature processing and are therefore not applicable to the type of polymeric substrate we have used. Nevertheless, there is a substantial literature that describes the change of crystal habit with doping of calcium phosphates, thus it may be possible to address this question by changing the topography of the calcium phosphate growth layer by the inclusion of a series of dopants. Given a sufficiently large variant series, it may then be possible to distinguish between the relative effects of surface topography and the composition of the growth layer.

P. Bongrand: The authors suggest that their model is a good starting point for further investigation: do they mean that it is possible to unravel the steps leading to chronic inflammation in vivo, or would they suggest to deposit different cell populations on their half coated structures in vitro, in order to determine the molecular and cellular requirements for FBGC formation?

Authors: We do think that a model such as this can be used to interrogate the inflammatory process in vivo. However, the approach we would adopt would be to modify the scaffold and/or scaffold coating and monitor the change in biological response, rather than by seeding differing cell populations on the scaffolds prior to implantation. 